\title{
Estudio paleontológico de los bivalvos (Mollusca) del Tortoniense superior de Arroyo Trujillo, Cantillana (Sevilla)
}

\author{
Joaquín CÁRDENAS ${ }^{*}$, Ildefonso BAJO² \& M. Vicente MAESTRE ${ }^{2}$ \\ ${ }^{1}$ Plaza de España, 10, $1^{\circ}$ D, 41702 Dos Hermanas, Sevilla; jcardecar@gmail.com \\ ${ }^{2}$ Museo de Alcalá de Guadaíra, Sección de Paleontología, c/ Juan Pérez Díaz s/n, 41500, Alcalá de Guadaíra, Sevilla \\ * Corresponding author
}

Cárdenas, J., Bajo, I. \& Maestre, M.V. 2017. Estudio paleontológico de los bivalvos (Mollusca) del Tortoniense superior de Arroyo Trujillo, Cantillana (Sevilla). [Palaeontological study of the bivalves (Mollusca) of the late Tortonian of Arroyo Trujillo, Cantillana (Sevilla)]. Spanish Journal of Palaeontology, 32 (2), 367-386.

\section{RESUMEN}

En este trabajo se actualiza la estratigrafía y se estudia la comunidad de bivalvos fósil del yacimiento Arroyo Trujillo (Cantillana, Sevilla) en la zona central de la Cuenca del Guadalquivir. Se ha realizado una nueva columna estratigráfica, y en ella se diferencian cuatro tramos correspondientes al Complejo Basal Transgresivo del Tortoniense superior. Han sido identificados 132 taxones de bivalvos, diez de los cuales se citan por primera vez en la península Ibérica. Se analiza la distribución de estas especies en los diferentes tramos estratigráficos del yacimiento. Esta comunidad de bivalvos se ha comparado con las de otros yacimientos, corroborando la similitud con el yacimiento de Cacela, Portugal. El estudio de las especies fósiles nos permite conocer condiciones de salinidad normal y clima subtropical-tropical.

Palabras clave: Cuenca del Guadalquivir, Arroyo Trujillo, Tortoniense superior, Complejo Basal Transgresivo (CBT), Bivalvia.

\begin{abstract}
In this work, the stratigraphy of Arroyo Trujillo (Cantillana, Sevilla) site, in the Guadalquivir Basin, is updated and the fossil bivalve community is studied. A new stratigraphic section has been made, differentiating four sections encompassed in the Transgressive Basal Complex of the upper Tortonian. One hundred and thirty two bivalves have been identified, 10 out of them are mentioned for the first time in the Iberian Peninsula. The distribution of these species in different stratigraphic sections is analysed. The community of bivalves has been compared with other sites, corroborating the similarity with Cacela site (Portugal). The fossil content suggests normal salinity conditions and subtropical-tropical climate.
\end{abstract}

Keywords: Guadalquivir Basin, Arroyo Trujillo, upper Tortonian, Transgressive Basal Complex (BTC), Bivalvia. 


\section{INTRODUCCIÓN}

Durante los diferentes eventos que constituyeron el proceso transgresivo ocurrido en la Cuenca del Guadalquivir durante el Tortoniense superior, se fueron depositando en su margen norte materiales de diversa naturaleza (Pendón et al., 2001; Abad, 2007). En la provincia de Huelva, el conjunto de dichos materiales fue denominado como Formación Calcarenitas de Niebla (Civis et al., 1987). Posteriormente, en un estudio más amplio, que tiene en cuenta que estos materiales basales cambian lateralmente de facies, y éstas no son siempre carbonatadas, se cambió dicho nombre a Formación Niebla (Baceta \& Pendón, 1999). Estudios posteriores realizados en el mismo margen norte de la zona central de la Cuenca (provincias de Sevilla y Huelva) a partir de estratigrafía secuencial, asignaron al conjunto de los materiales basales el nombre de Complejo Basal Transgresivo (CBT) (Pendón et al., 2001; Abad, 2002).

En un sentido amplio, estos materiales se pueden considerar como la parte inferior y media del cortejo sedimentario transgresivo (TST - Transgresive system tract) que rellenó la Cuenca del Guadalquivir (González Delgado \& Civis, 2000).

El CBT se extiende por el borde septentrional de la Cuenca del Guadalquivir formando una franja muy irregular en la que se localizan frecuentes yacimientos fosilíferos. De entre ellos, destacan los márgenes del Arroyo Trujillo, en el término municipal de Cantillana, al noreste de Sevilla, tanto por la variedad de especies, como por el moderado a buen estado de conservación de las mismas.

Fue Arias de Olivarrieta (1918) quien señaló por primera vez la presencia de fósiles en la zona en un informe enviado a la Real Sociedad Española de Historia Natural en 1918. En dicho informe, este autor refiere una lista de 32 moluscos, de los cuales 13 son bivalvos (Tabla 1) y los atribuyó al Plioceno. Posteriormente, Gavala (1927), en la realización de la hoja de Cantillana para el mapa Geológico de España, asigna a este yacimiento una edad Tortoniense (Mioceno), reconoce a su colega Fernández Iruela como redescubridor del yacimiento fosilífero y ofrece una lista de 13 bivalvos los cuales no coinciden exactamente con las especies dadas por Olivarrieta. Wenz (1942) estudia la zona y amplía la lista de bivalvos a 23 especies, asignando el yacimiento al Vindoboniense (Mioceno). Civis et al. (1990) realizan un amplio estudio del yacimiento incluyendo un estudio micropaleontológico del mismo. Citan 74 especies de moluscos de los cuales 34 son bivalvos (Tabla 1). Estos autores datan los materiales como Tortoniense superiorMessiniense (Mioceno).

Tabla 1. Relación de bivalvos dadas por autores anteriores que han estudiado el yacimiento.

\begin{tabular}{|c|c|c|c|}
\hline Olivarrieta (1918) & Gavala (1927) & Wenz (1942) & Civis et al. (1990) \\
\hline Meretrix italica & Callista pedemontana & Nucula mayeri & Nucula (N.) sp. \\
\hline Venus multilamella & Venus nux & Leda (Lembulus) fragilis & Nuculana (Saccella) fragilis \\
\hline Venus sp. & Lutraria lutraria & $\operatorname{Arca}($ A.) diluvii & Anadara (A.) diluvii \\
\hline Lutraria elliptica & Arca praecorbuloides & Glycymeris $(G$.) pilosa inflata & Glycymeris $(G$.) inflata \\
\hline Arca fichteli & Pectunculus gaditanus & Amussium (A.) cristatum & Glycymeris (G.) sp. \\
\hline Petunculus pilosus & Cardita rudista & Pecten (P.) benedictus & Amussium cristatum \\
\hline Solen vagina & Cardita jouanneti & Pecten (Amussiopecten) sp. & Chlamys (Aequipecten) scabrella \\
\hline Cardita bollenensis & Ostrea digitalina & Ostrea (Crassostrea) gingensis & Pecten (Amussiopecten) sp. \\
\hline Cardita jouanneti & Ostrea plicatula & Ostrea (O.) digitalina & Pecten (Flabellipecten) fraterculus \\
\hline Cardita sp. & Cardita crassa & Begina (Glans) aculeata globulina & Crassostrea gryphoides \\
\hline Cardium hoernesianum & Cardium hians & Venericardium (Megacardita) jouanneti & Ostrea (O.) edulis lamellosa \\
\hline Laevicardium oblongum & Cardium discrepans & Phacoides (Linga) columbella & Linga (L.) columbella \\
\hline \multirow[t]{8}{*}{ Pecten sp. } & Venus brochii & Lucina (Loripinus) fragilis & Myrtea (M.) spinifera \\
\hline & Venus plicata & Myrtea (M.) spinifera & Lucinoma borealis \\
\hline & & Cardium (Parvicardium) papillosum & Anodontia (Loripinus) fragilis \\
\hline & & Cardium (Ringicardium) hians rectum & Glans (C.) aculeata rhomboidea \\
\hline & & Macrocallista (Paradione) italica & Megacardita jouanneti \\
\hline & & Macrocallista (Paradione) erycinoides & Cardium (Bucardium) hians \\
\hline & & Meretrix (Cordiopsis) cf. islandicoides & Acanthocardia (A.) paucicostata \\
\hline & & Chione (Ventriculoidea) multilamella & Plagicardium (Papillicardium) papillosum \\
\hline
\end{tabular}




\begin{tabular}{|c|c|c|c|}
\hline Olivarrieta (1918) & Gavala (1927) & Wenz (1942) & Civis et al. (1990) \\
\hline & & Spisula (S.) subtruncata triangula & Laevicardium (L.) sp. \\
\hline & & Fabulina (Oudardia) compressa & Tellina (Oudardia) cf. compressa \\
\hline & & Aloidis (Varicorbula) gibba & Donax (Cuneus) cf. intermedia \\
\hline & & & Gari (Psammobia) fervensis \\
\hline & & & Venus (Ventricoloidea) multilamella \\
\hline & & & Pitar (P.) rudis \\
\hline & & & Callista (C.) italica \\
\hline & & & Callista (Costacallista) erycinoides \\
\hline & & & Pelecyora brocchii \\
\hline & & & Dosinia (Asa) lupinus \\
\hline & & & Clausinella cf. basteroti \\
\hline & & & Timoclea (T.) ovata \\
\hline & & & Corbula (Varicorbula) gibba \\
\hline & & & Corbula (Caryocorbula) revoluta \\
\hline
\end{tabular}

Tabla 1. (Continuación)

En un estudio comparativo realizado entre los diferentes yacimientos del Neógeno portugués, español y marroquí se cita el de Arroyo Trujillo (González Delgado et al., 1995). Posteriormente el primero de estos autores ha estudiado la ciclicidad estacional en concha de Megacardita jouanneti en los yacimientos de Cacela (Portugal) y Arroyo Trujillo (España) (González Delgado \& Civis, 2000).

En las últimas décadas se han realizado movimientos de tierra en la zona alta de los márgenes de Arroyo Trujillo con fines agrícolas y parcelarios, lo que ha permitido una mejor observación y estudio de los afloramientos. El objetivo de este trabajo es ampliar el conocimiento de las características geológicas y paleontológicas del yacimiento dando a conocer los 132 taxones de bivalvos identificados en los diferentes tramos hasta la fecha.

Por el elevado valor científico de este yacimiento clásico, Arroyo Trujillo ha sido catalogado entre los once puntos de mayor interés paleontológico del Neógeno de la provincia de Sevilla (Martínez Graña et al., 2016).

\section{LOCALIZACIÓN GEOGRÁFICA Y GEOLOGÍA}

La Cuenca del Guadalquivir es una larga depresión localizada al sur de la Península Ibérica a través de la cual se comunicaban las aguas del Océano Atlántico con las del Mediterráneo durante gran parte del Mioceno (Sanz de Galdeano \& Vera, 1991; Martín et al., 2001). Está limitada al norte por materiales Paleozoicos, al sur y al este por la Cordillera Bética y al suroeste se encuentra abierta al océano Atlántico a través del Golfo de Cádiz (Fig.1).
El yacimiento de Arroyo Trujillo se localiza a pocos kilómetros de la localidad de Cantillana, al norte de la provincia de Sevilla, y corresponde, por tanto, al margen norte de la Cuenca del Guadalquivir (Fig. 1). El cauce de este arroyo discurre, en su parte alta, sobre materiales del Carbonífero (Wagner \& Álvarez Vázquez, 2010), en su zona media sobre depósitos del Tortoniense superior (materiales detríticos del CBT), y en su zona baja, camino ya de la desembocadura en el Guadalquivir, sobre margas beige y grises pertenecientes a la unidad Formación Écija (Verdenius, 1970), equivalente a Arcillas de Gibraleón (Civil et al., 1987) de edad Tortoniense superiorMessiniense (Fig. 1).

Los depósitos del Tortoniense superior sobreyacen al sustrato Paleozoico del Carbonífero, formados por areniscas y conglomerados rojos de génesis deltaica.

Se han levantado dos columnas estratigráficas formadas por diferentes secuencias que corresponden a dos secciones del yacimiento separadas entre sí unos 1.000 m (Fig. 2). La sección $\mathrm{B}$ es la más representativa del yacimiento en lo que a bivalvos fósiles se refiere. Aunque en ella no aflora el muro de la formación ni el contacto con el zócalo, sí han podido ser observados en la sección A.

La sección A (Fig. 2) corresponde a la zona alta del cauce y presenta, de muro a techo, tres de los cinco tramos diferenciados en el yacimiento:

1) Tramo I. Presenta un subnivel no superior a $0,5 \mathrm{~m}$ de materiales procedentes del zócalo y muy removidos. En discordancia sobre éste se superponen $6 \mathrm{~m}$ de arenas marrones con gravillas cuyos clastos, generalmente redondeados, oscilan entre 2 y $10 \mathrm{~mm}$ de diámetro. Ambos materiales poco seleccionados. Continúan $3 \mathrm{~m}$ de arenas amarillas con una ligera laminación en la base. 


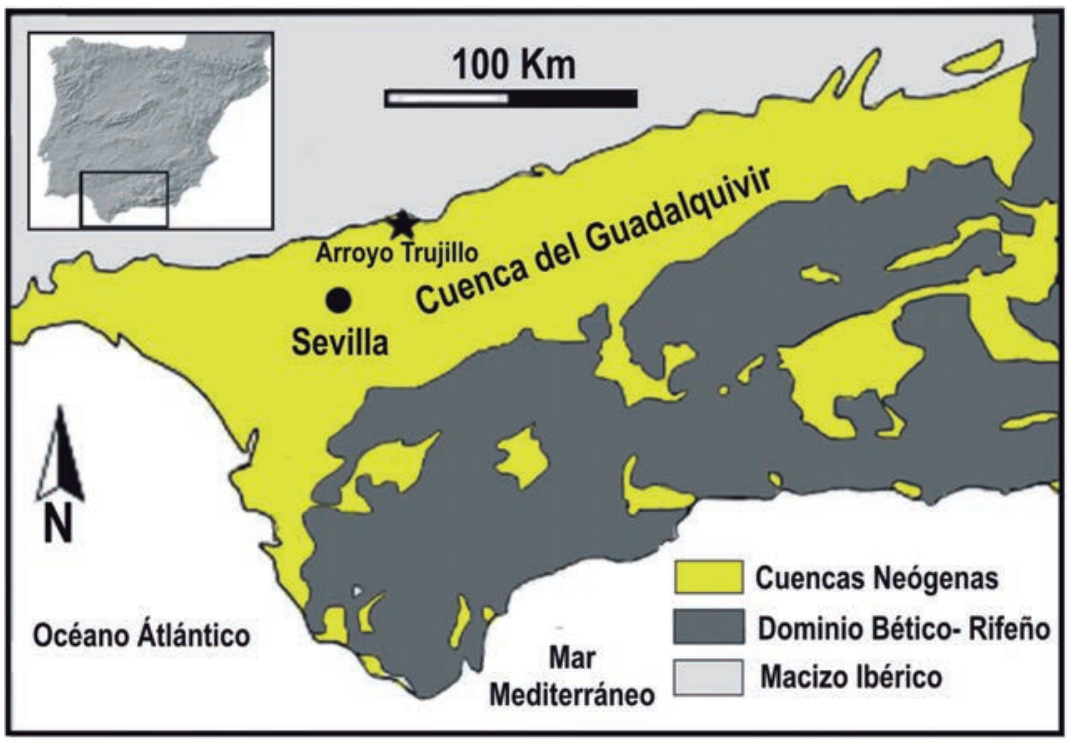

Figura 1. Situación geológica y geográfica de la zona estudiada.

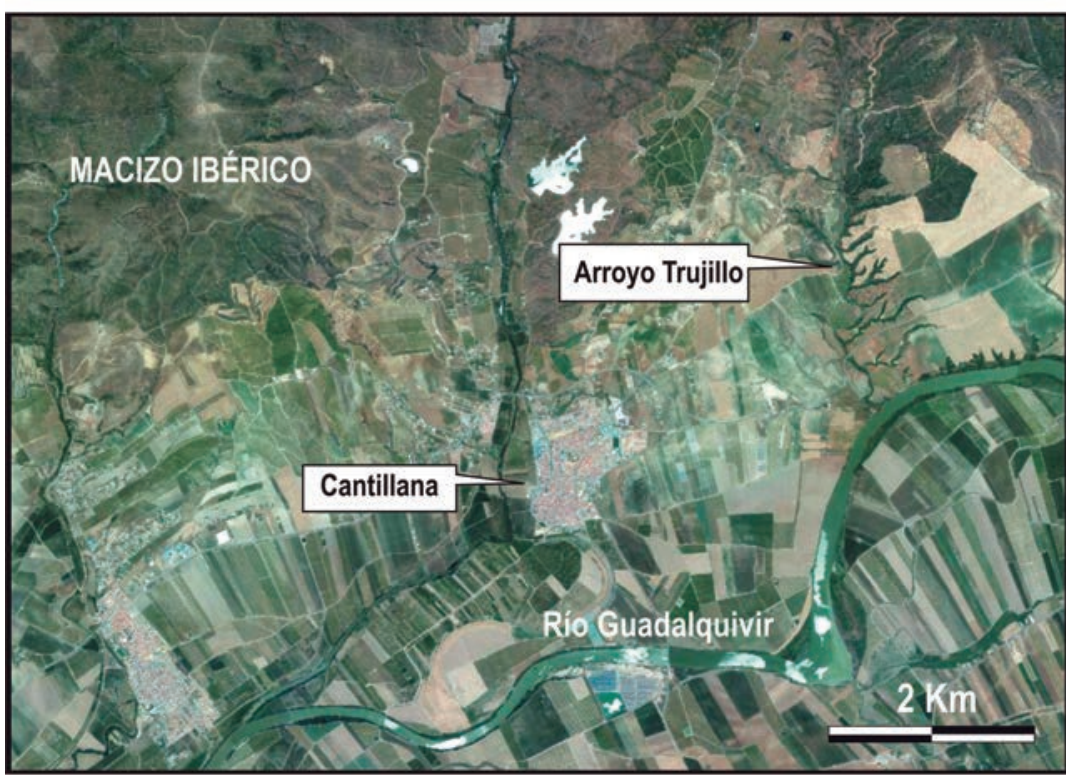

2) Tramo II. Comienza con $5 \mathrm{~m}$ de arenas gruesas con gravillas donde se intercalan tres niveles de conglomerados: a) el primero de ellos de poco espesor y con clastos sueltos; b) el segundo con un espesor de 0,5 m, muy cementado y con abundantes Crassostrea gryphoides; y, c) el tercero, también cementado, solo presenta algunos fragmentos de ostreidos muy dispersos y, generalmente, no identificables taxonómicamente.

3) Tramo III. Sobre el tramo anterior se depositan, finalmente, 20-30 $\mathrm{m}$ de arenas limosas de color beige con intercalaciones de bloques de arenisca de forma y tamaño variables. En este tramo los macrofósiles son muy escasos y dispersos. Entre ellos destaca Aequipecten scabrella.

La sección B (Fig. 2) está situada más hacia el interior de la cuenca. Presenta, de muro a techo, los cuatro tramos superiores de los cinco diferenciados en el yacimiento:
1) Tramo II. Formado por $1,5 \mathrm{~m}$ de arenas marrones fuertemente cementadas y con una elevada concentración de Heterostegina gomez-angulensis. Dicha concentración de heterosteginas decrece a techo del tramo y entre ellas se localizan algunos restos de Crassostrea gryphoides y excepcionalmente de otras especies. Las secuencias subyacentes a este tramo hasta el zócalo no son visibles en esta sección. En la Figura 2 se marca la posible correlación entre ambas secciones.

2) Tramo III. Formado por unos $13-15 \mathrm{~m}$ de arenas limosas marrones. En ellas, en las que las heterosteginas se encuentran muy dispersas, son frecuentes niveles de concentración de fósiles, acompañados de arenas algo más gruesas. Estos niveles de poca continuidad horizontal, tienen un espesor variable entre 15 a $30 \mathrm{~cm}$. A veces, por procesos de cementación postdiagénica, dan lugar a nódulos de formas muy irregulares que se hallan 


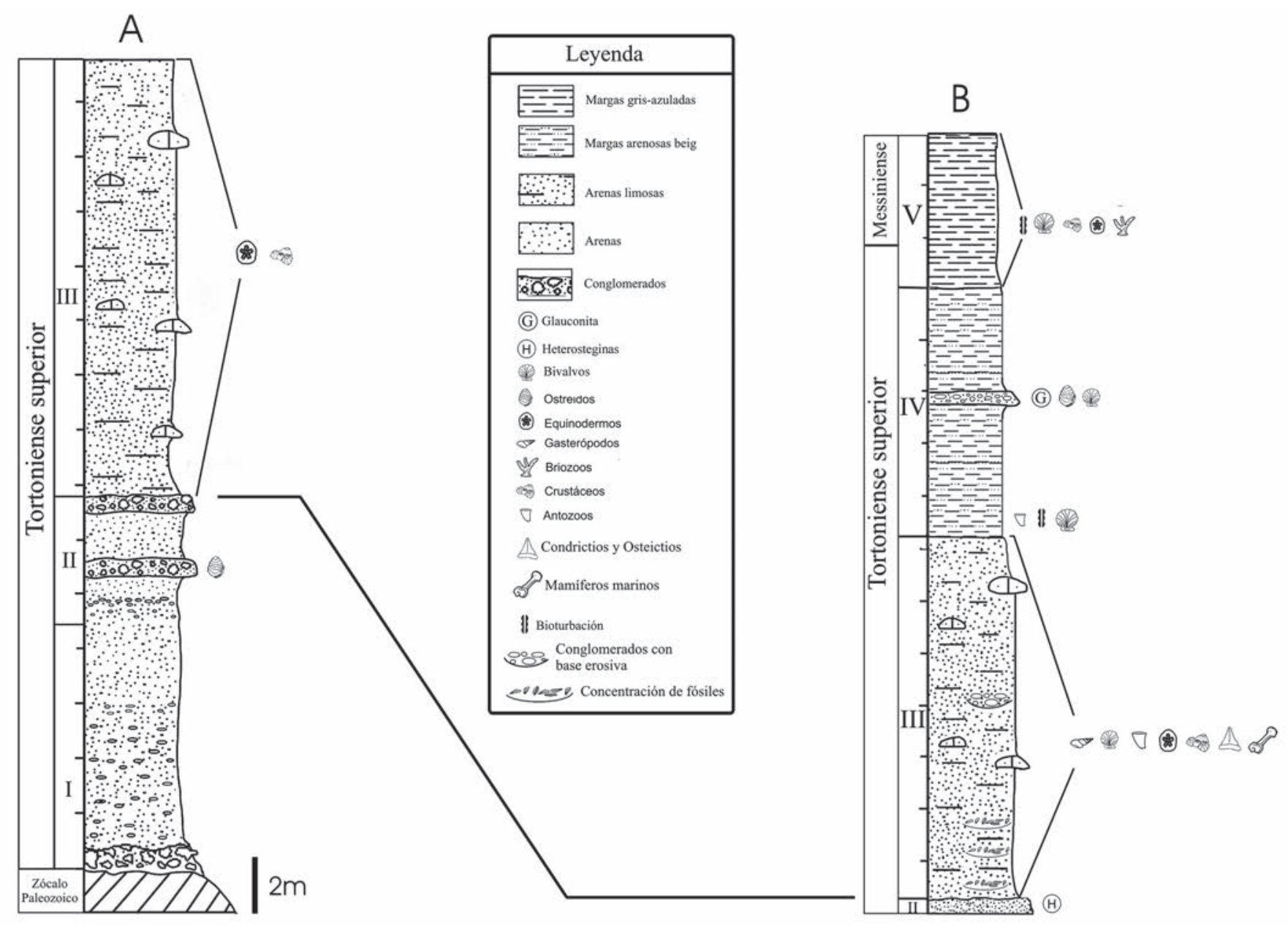

Figura 2. Columnas estratigráficas de Arroyo Trujillo (Cantillana). Se muestran dos secciones del yacimiento, A y B, correlacionadas.

intercalados en el sedimento. En la parte central de este tramo se localizan paleocanales de base cóncava erosiva rellenos de arenas y conglomerados de tamaño medio a grueso de color rojizo procedentes de la erosión del sustrato Carbonífero. Sobre éstos continúa el depósito de las arenas limosas marrones.

3) Tramo IV. Las arenas anteriores pasan progresivamente a 8-10 $\mathrm{m}$ de arenas finas margosas de color beige, intensamente bioturbadas en su base y con fósiles dispersos. Entre ellos suelen localizarse bivalvos con sus valvas articuladas. Intercalado en este tramo, próximo al techo y de forma discordante, se aprecia un nivel de arenas con abundante glauconita. Sobre estas arenas se dispone otro nivel de conglomerados con clastos de tamaño mediano. El conjunto no supera los $0,5 \mathrm{~m}$ de potencia y en él se encuentran fósiles fragmentados y desgastados.

4) Tramo V. Finalmente, las arenas margosas pasan de forma gradual a margas gris verdosas con una potencia visible de 5 a $7 \mathrm{~m}$ (observables en arroyadas cercanas). En la zona superior de estas margas se localiza el tránsito Tortoniense-Messiniense (Sierro y Flores, en Civis et al., 1990).

\subsection{Cronología}

Las primeras dataciones del yacimiento se realizaron por correlación estratigráfica y de acuerdo a la fauna de moluscos localizada en el mismo. Arias de Olivarrieta (1918) lo asigna al Plioceno, Gavala (1927) propone una edad de Mioceno superior (Tortoniense) y Wenz (1942) de Mioceno medio (Vindoboniense).

La datación más reciente está basada en foraminíferos planctónicos y nanoplancton (Sierro, y Flores, en Civis et al., 1990). El conjunto de especies identificadas permitió a estos autores englobar los materiales dentro de la secuencia deposicional B de Sierro (1985), coincidente con el ciclo 3,2 de Haq et al. (1987). Ambos elementos tienen como edad superior 7,5 Ma. Teniendo en cuenta que estos materiales pasan sin interrupción al Messiniense en la misma columna estratigráfica (Civis et al., 1990), y considerando que dicho paso está datado en 7,2 Ma, los tramos inferiores del CBT, donde se localiza la fauna estudiada en este trabajo, estarían comprendidos entre los 7,5 Ma y 7,2 Ma. 
Por otra parte, el yacimiento de Cacela (Portugal) ha sido datado en 8,2 Ma por la presencia de los taxones Discoaster berggrenni y Discoaster quinqueramus (Cachao, 1995). Si, tal como señalan algunos autores (González Delgado et al., 1995) los yacimientos de Cacela y Arroyo Trujillo son sincrónicos y además tenemos en cuenta que en Arroyo Trujillo también se han localizados los taxones Discoaster berggrenni y D. quinqueramus (Flores, en Civis et al., 1990), habría que considerar que la edad de los materiales del CBT se encontrarían comprendida entre 8,2 Ma y 7,2 Ma.

\section{MATERIAL Y MÉTODOS}

Se han realizado diversas visitas de trabajo a la zona, algunas de ellas coincidiendo con movimientos de tierra realizados en el lugar con fines agrícolas o parcelarios. En estas visitas se han llevado a cabo exhaustivas prospecciones visuales, con recogida de ejemplares, tanto en materiales removidos como en los nuevos cortes expuestos. Se han recogido más de 20 muestras de sedimento de diferentes lugares previamente seleccionados. Se ha tomado al menos una muestra en cada tramo. En el tramo III se han tomado de 2 a 3 muestras de cada nivel de concentración de fósiles y al menos 5 muestras en las arenas sin concentración de fósiles. En total han sido analizados más de $100 \mathrm{~kg}$ de sedimento con el objeto de localizar micromoluscos. La mayoría de las muestras se han reblandecido en abundante agua y posteriormente han sido cribadas con un tamiz de luz de malla $0,5 \mathrm{~mm}$. Una vez secos, los elementos superiores a $0,5 \mathrm{~mm}$ han sido analizados con la lente binocular y de forma previa a la extracción de los moluscos de pequeño tamaño, se han realizado conteos de conchas y bioclastos en un tercio de todas las muestras para determinar la abundancia de los diferentes grupos.

Otras muestras formadas por terrones de sedimento y con indicios de fósiles en su interior han sido disgregadas mediante el uso de aguja enmangada y pincel húmedo para recuperar los ejemplares de fósiles de mayor fragilidad.

Es frecuente la presencia de nódulos de sedimentos muy cementados cuyo núcleo lo constituyen los propios fósiles. Algunos de estos nódulos han sido fragmentados pudiendo recuperar de su interior algunos ejemplares fósiles.

Para la identificación de las especies y posterior clasificación taxonómica han sido consultados, entre muchos otros, los trabajos de Sacco (1897-1898), CerulliIrelli (1908), Cossmann \& Peyrot (1911), Kautsky (1939), Venzo \& Pelosio (1963), Marasti (1973), Malatesta (1974), Andrés (1982), Studenka (1986) y Lozano Francisco (1997). Para la asignación de los géneros a las diferentes familias se ha seguido a Millard (1997) con algunas adaptaciones. Para aquellos taxones que cuentan con representación en la actualidad se han consultado Bouchet \& Rocroi (2010), Sabelli et al. (1990-92) y Gofas et al. (2011), además de las bases de datos "online" CLEMAM (http.//www.somali.asso.fr/clemam/index.elemam.html) y WoRMS (www.marinespecies.org).

Todo el material forma parte de la colección de referencia de los autores. Una vez concluidos los diferentes trabajos previstos sobre otros grupos fósiles del yacimiento (alguno en preparación) será depositado en el Museo de Geología de la Universidad de Sevilla.

\section{ESTUDIO DE LOS BIVALVOS DEL YACIMIENTO}

Se han identificado un total de 132 taxones de bivalvos (Tabla 2). De ellos, en 125 se ha llegado a nivel de especie. De estas especies, 93 son citas nuevas en el yacimiento y 10 de ellas son primeras citas en la Península Ibérica. El conjunto taxonómico corresponde a 43 familias, de las cuales las 12 con mayor número de especies representadas constituyen el $64,66 \%$ de todas las especies identificadas en el yacimiento. Las otras 31 familias representadas abarcan el $35,33 \%$ de la totalidad de las especies (Fig. 3). La familia más representativa es Veneridae con 16 especies. Del número total de taxones identificados, 7 se han dejado con nomenclatura abierta. Dos de ellos, Hemilepton sp., Thracia sp. y otro citado como Donacilla aff. cornea, pueden ser especies aún no descritas cuyo estudio se retomará en trabajos próximos. De entre las especies identificadas destacan varias por su peculiar distribución: 1) Digitaria convergens, citada únicamente en los yacimientos de Cacela y Cantillana; 2) Arcopagia ventricosa, citada en el Mioceno inferior y medio de la costa atlántica (Cossman \& Peyrot, 1911), en el Tortoniense de Cacela y Cantillana y más reciente en el Pleistoceno de Torrente Arda (Italia) (Brunetti \& Vecchi, 2005); y, 3) Donax venustus, citada únicamente en Cacela y Cantillana para el Tortoniense superior y posteriormente se extiende por las cuencas atlántica y mediterránea donde tiene presencia en la actualidad. Respecto del conjunto de las especies en el yacimiento, los taxones más constantes en todas las muestras estudiadas, por su presencia y cantidad de ejemplares, son microbivalvos de un tamaño comprendido entre 3 y $10 \mathrm{~mm}$, correspondientes a las especies Saccella conmutata, Gouldia minima, Microloripes dentatus, Parvicardium minimum, Crassatina concentrica y Cyclocardia scalaris, seguidos, con menor número de ejemplares de Nucula nucleus, Limea strigilata, Lucinaxinus reticulatus, Lucina agassizi, Similipecten similis, Timoclea ovata y Corbula gibba (Fig. 4). 


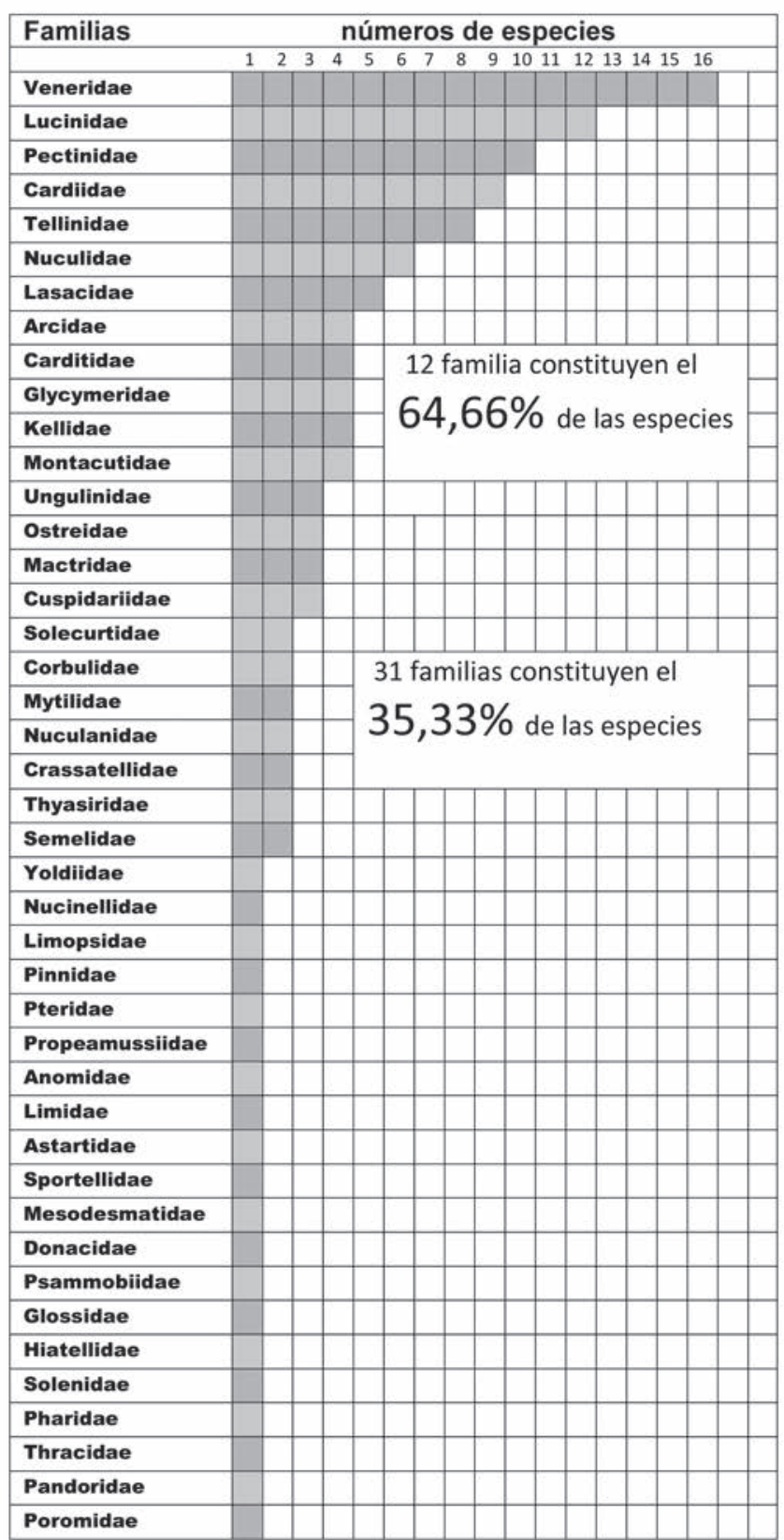

Figura 3. Familias de bivalvos en el yacimiento y número de especies que las representan.

Comparando las comunidades de bivalvos de los yacimientos Arroyo Trujillo y Cacela (Portugal) es posible observar similitudes, como que las cinco familias más representativas coinciden en ambos yacimientos y que la familia Veneridae es mayoritaria (Santos \& Mayoral, 2007). El yacimiento de Arroyo Trujillo comparte 76 especies de bivalvos $(60,8 \%)$ con el yacimiento de Cacela (Pereira Da Costa, 1903-1904; Santos \& Mayoral, 2007). Aplicando el índice de Sorensen a las dos comunidades nos indica $69 \%$ de similitud entre ambas. Otros yacimientos de la fachada atlántica que se han comparado con Arroyo
Trujillo han sido: 1) Cuenca de Sais (Marruecos), del Tortoniense-Messiniense, donde se han reconocido 48 bivalvos, de los cuales 32 especies coinciden con las de Arroyo Trujillo (Lauriat-Rage et al., 1997); y, 2) costa de Caparica (Foz de Rego-Portugal), del Tortoniense inferior, con 39 bivalvos, de los que 28 han sido citados en Arroyo Trujillo (Mocho et al., 2010).

Del conjunto de especies de Arroyo Trujillo, 73 especies (que representan el 58,5\% del total) han sido citadas en el Tortoniense de la cuenca mediterránea (Sacco, 18971898; Cerulli-Irelli, 1908; Venzo \& Pelosio, 1963; Robba, 1968; Marasti, 1973; Acuña, 1978; Lauriat-Rage et al., 1997; Martínez-Lázaro, 2004). Dicho porcentaje aumenta considerablemente si se consideran las citas realizadas para el Mioceno superior (Tortoniense-Messiniense). Otras especies localizadas en este yacimiento, Ennucula corbuloides, Ctena decorata, Gonimyrtea gracilis, Thyasira succisa, Felaniella brevifulcrata, Planikellya punctata, Pseudopythina macandrewi, Spisula inaequilatera, Poromia granulata (Fig. 5), no han sido citadas en la cuenca mediterránea pero si en la fachada atlántica, sobre todo en el Plioceno de la cuenca del Mar del Norte (Bélgica, Holanda, Dinamarca, Alemania). Algunas de ellas aún están presente en el Océano Atlántico (Nyst, 1878-1881; Nyst \& Westendorp, 1839; Cossmann \& Peyrot, 1911; Janssen \& Van Der Slik, 1971; Wesselingh \& Moerdijk, 2007), y otras lo hacen, actualmente, en el Mediterráneo (Gofas et al., 2011).

\subsection{Fauna acompañante}

La realización de este trabajo ha permitido obtener abundante material perteneciente a otros grupos taxonómicos. Destacan los gasterópodos, que junto con los escafópodos, superan los 180 taxones identificados hasta el momento. Los grupos antozoos, crustáceos y equinodermos están bien representados y los vertebrados lo están con frecuentes otolitos y dientes de osteíctios y dientes de condrictios. También son frecuentes en el yacimiento restos óseos de mamíferos marinos (cetáceos). Otros grupos, sin embargo, se encuentran escasamente representados. Es el caso de braquiópodos, representados únicamente por Criptopora lovisati, anélidos, representados por Ditrupa arietina, y briozoos, de los que salvo la frecuente Discoporella umbellata, sólo se localizan escasos fragmentos de colonias.

\subsection{Distribución de las especies en los diferentes tramos estratigráficos. Tafonomía y paleobiología}

Las buenas condiciones de fosilización que ha presentado el yacimiento y posterior conservación de conchas 
Tabla 2. Tabla de las especies de bivalvos identificadas en el yacimiento de Arroyo Trujillo (Cantillana) correspondiente al Tortoniense superior. Se indica su distribución en los diferentes medios marinos, su distribución estratigráfica y su frecuencia en el yacimiento. Para la frecuencia se ha seguido el siguiente baremo: Rara (R): 1 ejemplar en todo el material analizado; Escasa (E): hasta 5 ejemplares; Frecuente (F): hasta 20 ejemplares; Abundante (A): hasta 50 ejemplares; Muy abundante (MA): más de 50 ejemplares.

\begin{tabular}{|c|c|c|c|c|c|c|c|}
\hline Especies bivalvos & 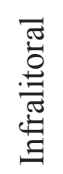 & 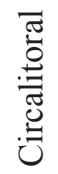 & 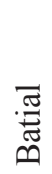 & 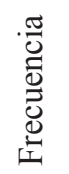 & $\begin{array}{l}\stackrel{0}{0} \\
\stackrel{0}{0} \\
\stackrel{0}{\Sigma}\end{array}$ & $\begin{array}{l}\stackrel{0}{0} \\
\stackrel{0}{0} \\
\stackrel{0}{0}\end{array}$ & 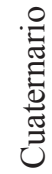 \\
\hline \multicolumn{8}{|l|}{ Nuculidae } \\
\hline \multicolumn{8}{|l|}{ Nucula nitidosa } \\
\hline \multicolumn{2}{|l|}{ Nucula nucleus } & \multicolumn{6}{|c|}{ Nucula placentina } \\
\hline \multicolumn{8}{|l|}{ Nucula sulcata } \\
\hline \multicolumn{8}{|l|}{ Ennucula corbuloides } \\
\hline \multicolumn{8}{|l|}{ Leionucula laevigata } \\
\hline \multicolumn{8}{|l|}{ Nuculanidae } \\
\hline \multicolumn{8}{|l|}{ Nuculana pella } \\
\hline \multicolumn{8}{|l|}{ Saccella conmutata } \\
\hline \multicolumn{8}{|l|}{ Yoldiidae } \\
\hline \multicolumn{8}{|l|}{ Yoldia nitida } \\
\hline \multicolumn{8}{|l|}{ Nucinellidae } \\
\hline \multicolumn{8}{|l|}{ Nucinella ovalis } \\
\hline \multicolumn{8}{|l|}{ Arcidae } \\
\hline \multicolumn{8}{|l|}{ Anadara diluvii } \\
\hline \multicolumn{8}{|l|}{ Anadara fichteli } \\
\hline \multicolumn{8}{|l|}{ Anadara pectinata } \\
\hline \multicolumn{8}{|l|}{ Bathyarca pectunculoides } \\
\hline \multicolumn{8}{|l|}{ Limopsidae } \\
\hline \multicolumn{8}{|l|}{ Limopsis anomala } \\
\hline \multicolumn{8}{|l|}{ Glycymerididae } \\
\hline Glycymeris bimaculata & & & & $\mathrm{E}$ & & & \\
\hline Glycymeris inflata & & & & $\mathrm{F}$ & & & \\
\hline Glycymeris insubrica & & & & $\mathrm{F}$ & & & \\
\hline Glycymeris sp. & & & & $\mathrm{R}$ & & & \\
\hline Mytilidae & & & & & & & \\
\hline Gibbomodiola adriatica & & & & $\mathrm{E}$ & & & \\
\hline Modiolus sp. & & & & $\mathrm{E}$ & & & \\
\hline Pinnidae & & & & & & & \\
\hline Atrina pectinata & & & & $\mathrm{E}$ & & & \\
\hline Pteriidae & & & & & & & \\
\hline Pteria phalaenacea & & & & $\mathrm{E}$ & & & \\
\hline Pectinidae & & & & & & & \\
\hline Oppenheimopecten revolutus & & & & $\mathrm{E}$ & & & \\
\hline Aequipecten radians & & $?$ & & $\mathrm{R}$ & & & \\
\hline Aequipecten scabrella & & & & A & & & \\
\hline Cristatopecten cristatum & & & & $\mathrm{F}$ & & & \\
\hline Talochlamys multistriata & & & & $\mathrm{F}$ & & & \\
\hline Flabellipecten fraterculus & & $-?$ & & $\mathrm{~F}$ & & & \\
\hline Flabellipecten solarium & 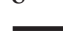 & & & $\mathrm{E}$ & & & \\
\hline Gigantopecten albinus & & & & $\mathrm{F}$ & & & \\
\hline Costellamussiopecten spinulosus & & & & $\mathrm{F}$ & & & \\
\hline Palliolum incomparabilis & & & & $\mathrm{F}$ & & & \\
\hline Propeamussiidae & & & & & & & \\
\hline Similipecten similis & & & & A & & & \\
\hline
\end{tabular}




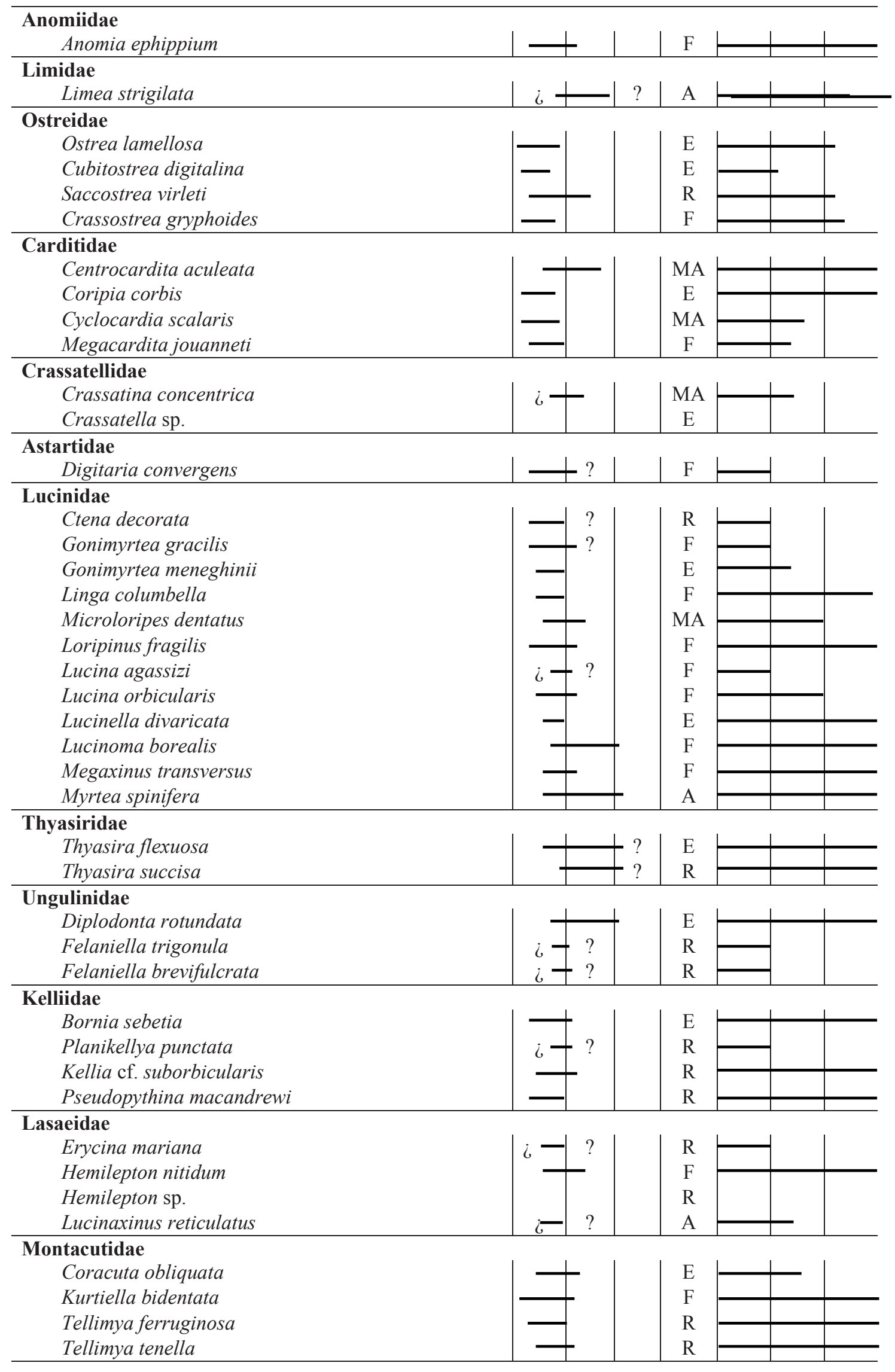




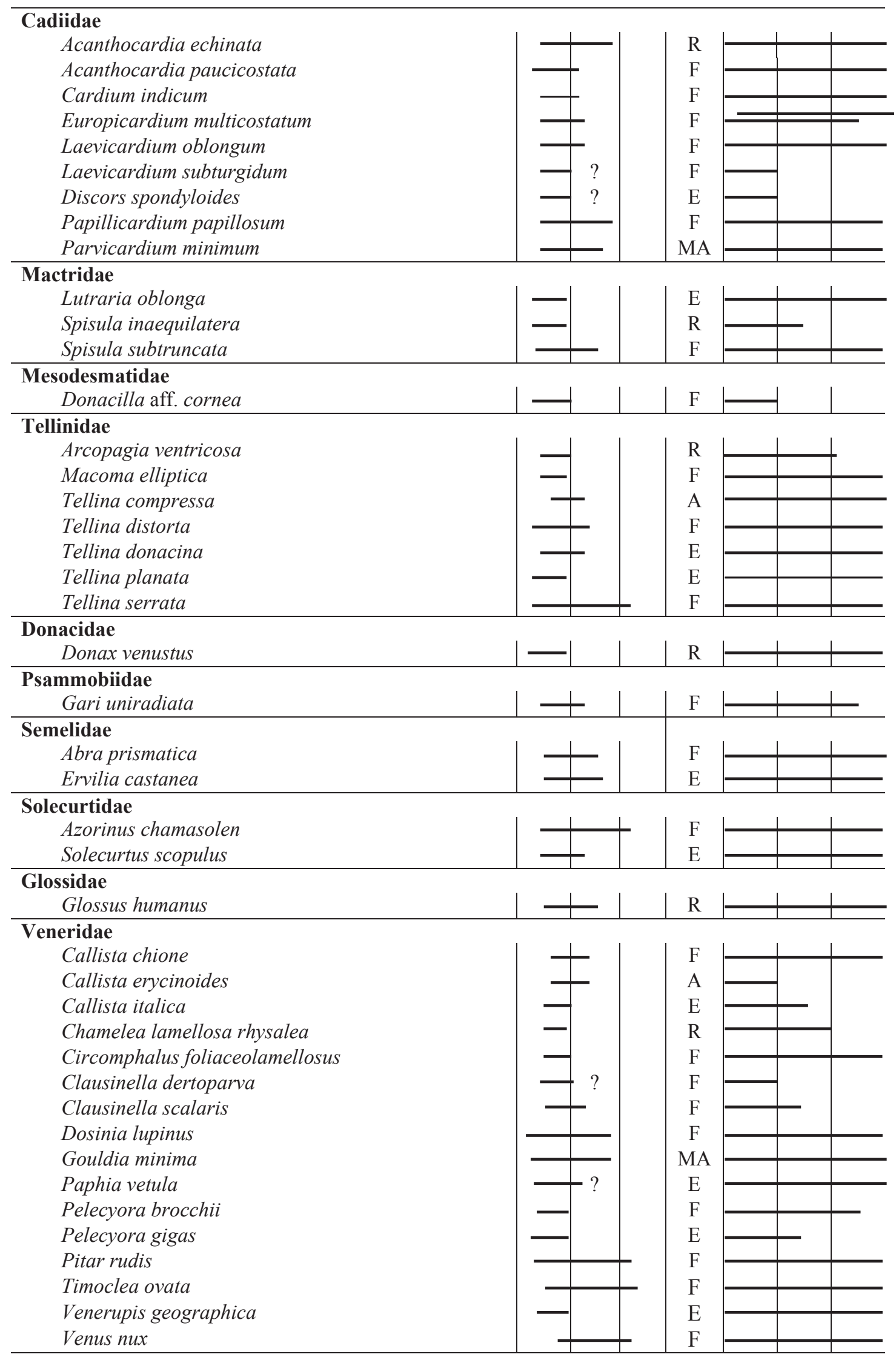




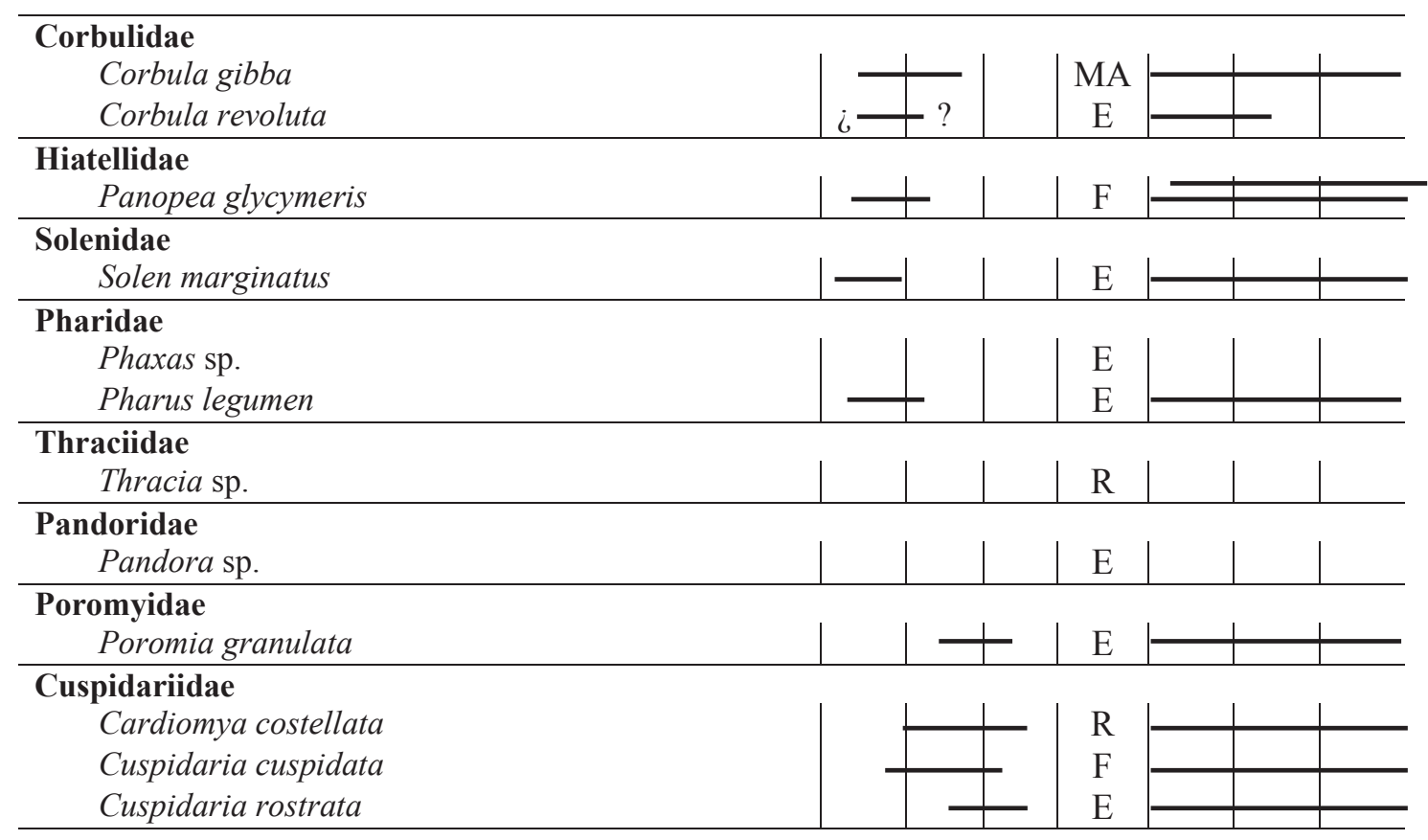

Tabla 2. (Continuación)

de moluscos, ha permitido la preservación de fósiles corporales no alterados, tanto de conchas calcíticas como aragoníticas.

Estas conchas fosilizadas, y por tanto las especies, se encuentran distribuidas en los diferentes tramos del yacimiento en función de la naturaleza del sedimento que lo compone, batimetría a la que se depositó dicho sedimento y transporte al que fueron sometidos los especímenes. Teniendo en cuenta esto, podemos comprobar la siguiente distribución:

1) Tramo I, formado por arenas y gravillas. Estériles en bivalvos.

2) Tramo II, de arenas gruesas y cantos rodados. Presentan, a veces, grupos de Crassostrea gryphoides con sus valvas articuladas, de tamaño grande junto a otros ejemplares juveniles. Ocasionalmente se localizan otras especies de ostreidos y pectínidos no identificables taxonómicamente por su fragmentación y desgaste.

3) Tramo III, de arenas limosas con niveles de concentración de fósiles.

Dichos niveles se interpretan como resultado de movimientos de oleaje y corrientes que en épocas de tormenta remueven cantidades grandes de sedimento los cuales quedan en suspensión hasta que, una vez estabilizada la agitación, vuelven a depositarse de modo granodecreciente (González Delgado et al., 1995). Durante estos episodios es posible que se destruyeran las trazas de bioturbación, así como ser causantes del arrastre de conchas de unas zonas a otras.
Las conchas fósiles que se localizan fundamentalmente en este tramo, y desde un punto de vista tafonómico, se pueden agrupar en los siguientes tres grupos con límites muy flexibles:

a) Ejemplares con valvas completas y frecuentemente articuladas. Estas, a veces, se presentan fragmentadas por procesos mecánicos posteriores a la fosilización. En sus caras externas no presentan erosión ni desgaste y los elementos ornamentales, como espinas o lamelas, se conservan en perfecto estado. Presentan frecuentes bioperforaciones (Oichnus simplex y $O$. paraboloides) producidas, en su mayoría, por gasterópodos carnívoros. Su hábitat posiblemente fuera el mismo donde quedaron enterrados y su transporte habría sido mínimo y ocasional. Entre las especies más representativas de este grupo se encuentran Callista erycinoides, Glycymeris insubrica, G. inflata, Centrocardita aculeata, Anadara diluvii, A. fichteli, Venus nux, Macoma elliptica, Megacardita jouanneti, Tellina compressa, Pelecyora brochii y, en menor proporción, Flabellipecten fraterculus y Panopea glycymeris. Junto a éstas se encuentran todas las especies de pequeño tamaño citadas anteriormente y las demás especies reconocidas en el yacimiento y no mencionadas en otros tramos.

b) Valvas completas y con frecuencia fragmentadas, pero con más del $50 \%$ de la valva. Corresponden a especies poco representadas en el yacimiento y pueden presentar más erosión o desgaste debido a un mayor transporte post morten desde zonas someras (infralitoral 


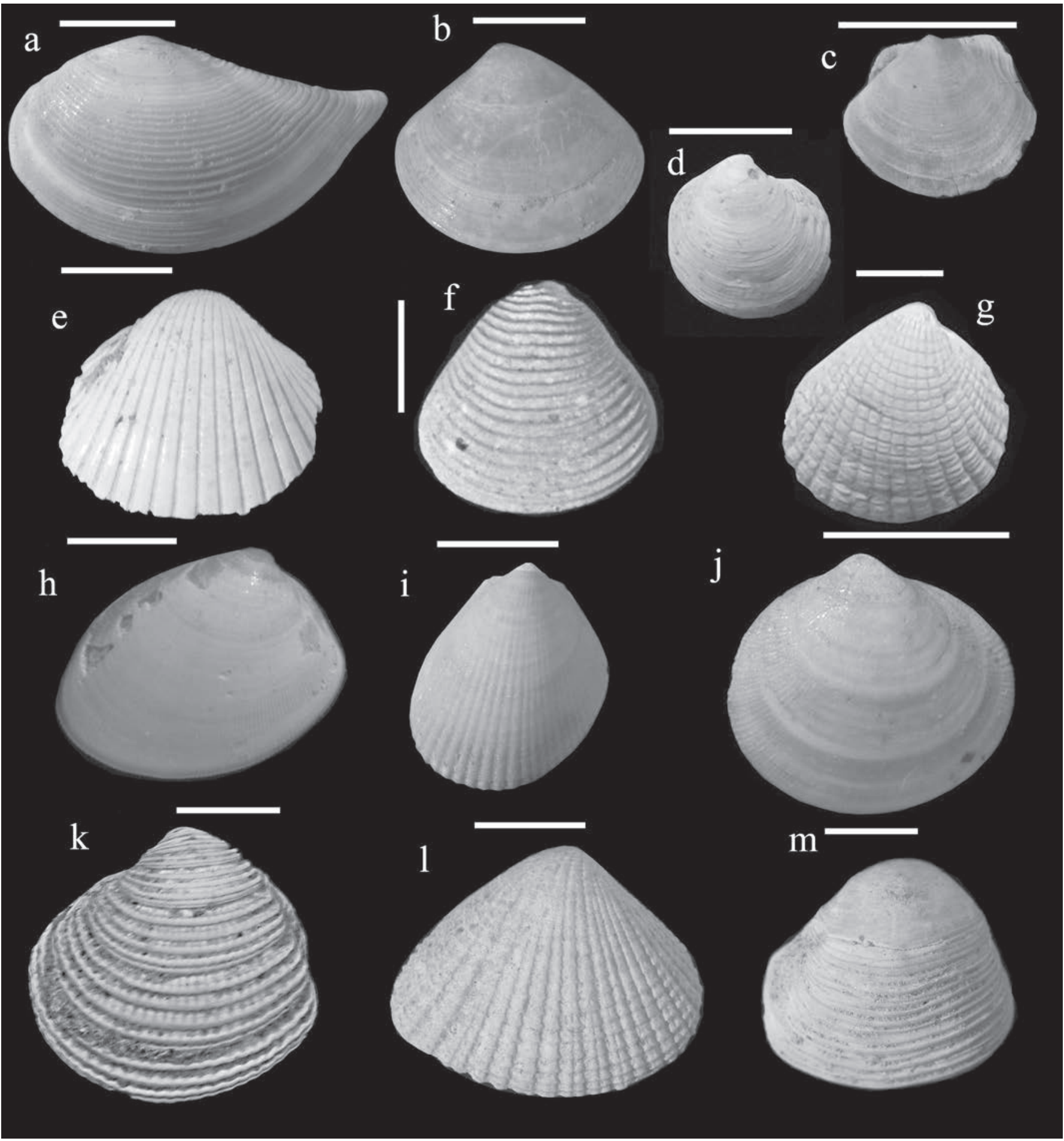

Figura 4. a) Saccella conmutata valva Izquierda. b) Gouldia minima valva izquierda. c) Similipecten similis valva izquierda. d) Microloripes dentatus valva derecha. e) Parvicardium minimum valva derecha. f) Crassatina concentrica valva derecha. g) Cyclocardia scalaris valva derecha. h) Nucula nucleus valva izquierda. i) Limea strigilata valva derecha. j) Lucinaxinus reticulatus valva derecha. k) Lucina agassizi valva izquierda. l) Timoclea ovata valva derecha. m) Corbula gibba valva derecha. Escala de las barras $=2,5 \mathrm{~mm}$.

interna), como Ostrea lamellosa, Cubitostrea digitalina y Saccostrea virleti, Aequipecten scabrella y A. radians, o desde zonas más profundas (circalitorales), como Bathiarca pectunculoides, Cardiomya costellata, Ennucula corbuloides, Leionucula laevigata, Poromia granulata, Cuspidarias cuspidata y Cuspidaria rostrata. El hábitat reconocido para este último grupo de especies suele ser la zona circalitoral o incluso para algunas la zona batial 


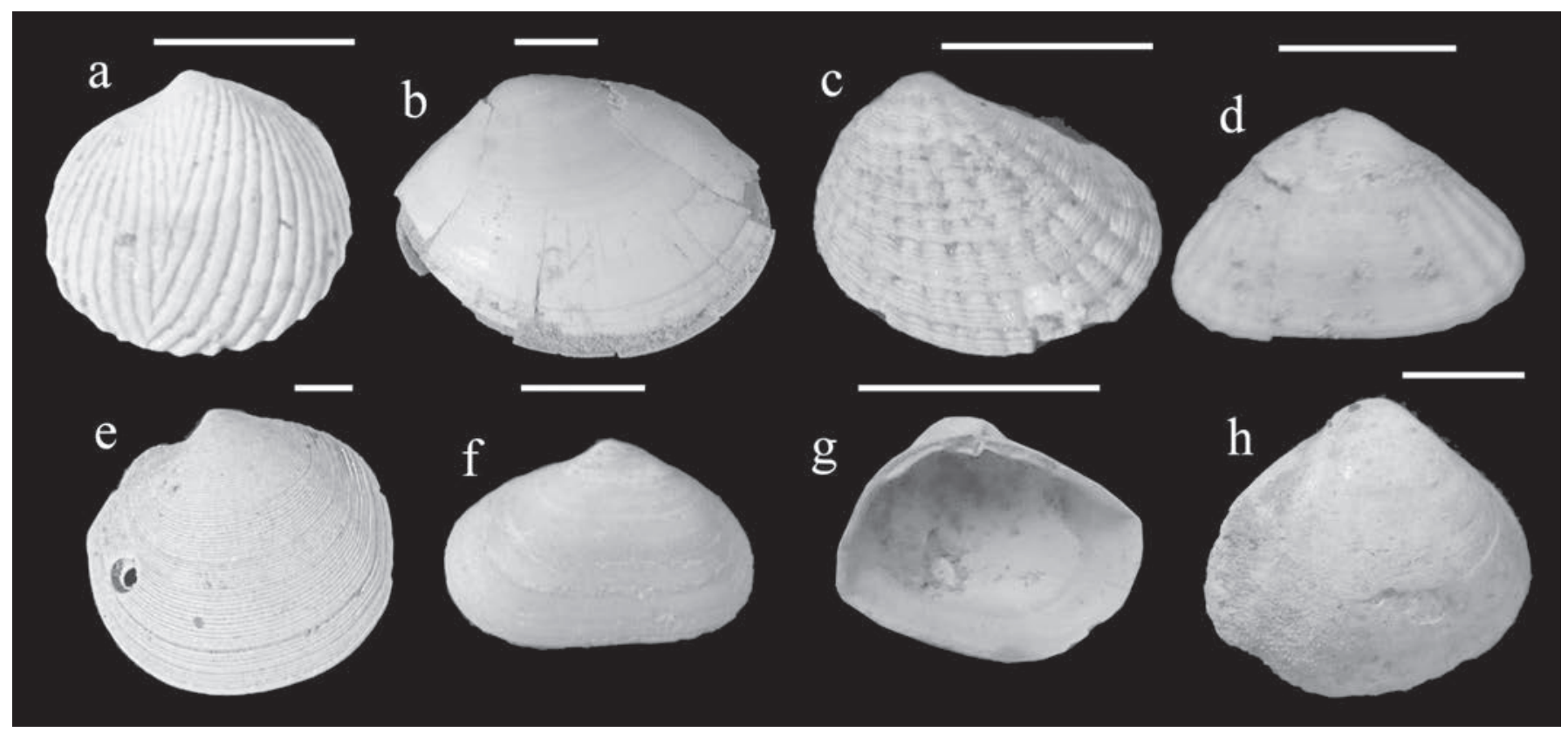

Figura 5. a) Digitaria convergens valva izquierda. b) Leionucula laevigata valva derecha. c) Ctena decorata valva derecha. d) Planikellia punctata valva derecha. e) Gonimyrtea gracilis valva izquierda. f) Pseudopytina macandrewi valva izquierda. g) Spisula inaequilatera valva izquierda, cara interna. h) Poromia granulata, valva derecha. Escala de las barras $=2,5 \mathrm{~mm}$.

(Allen \& Morgan, 1981; Janssen \& Krylora, 2014). Otras especies frecuentes en la zona infralitoral profunda y circalitoral somera serían Glossus humanus, Cristatopecten cristatum, Arcopagia ventricosa, Atrina pectinata, Limopsis anomala, Palliolum incomparabilis, Thyasira flexuosa y T. succisa (Figs. 6-8).

c) Conjunto de fragmentos, bioclastos de todas las especies donde se incluyen algunas conchas completas o semi completas, sobre todo de pequeño tamaño. Presentan bastante desgaste a causa de ser continuamente batidos por los movimientos de corrientes, mareas y/u oleajes.

Una característica general para todos los ejemplares de los tres grupos señalados anteriormente es la carencia total de bioincrustaciones de briozoos, crustáceos, anélidos u otros grupos debido, posiblemente, a permanecer éstos poco tiempo en la superficie del sedimento antes de ser enterrados.

4) Tramo IV, de arenas finas margosas de color beige. Tramo que, por su situación estratigráfica y por el incremento en especies de foraminíferos planctónicos (Sierro, en Civis et al., 1990), denota mayor profundidad del medio con abundante bioturbación. Los fósiles se encuentran muy dispersos y, con frecuencia, completos y articulados. Los infaunales suelen presentarse en posición de vida, es decir, articulados, con el plano de comisura vertical u oblicuo a la horizontal y su seno paleal en la parte superior de la concha. Se repiten muchas especies del tramo anterior como Callista ericynoides, Callista chione, Anadara diluvii y Saccella conmutata, junto a otros de escasa representación en el tramo anterior como son las especies epifaunales Cristatopecten cristatum y Costellamussiopecten spinulosus.

5) Tramo IV-a, nivel de arenas con glauconita y conglomerados. En él se localizan conchas de Gigantopecten albinus, Flabellipecten solarium, Aequipecten scabrella y fragmentos de Ostrea sp. Los ejemplares en este tramo están frecuentemente fragmentados y desgastados.

6) Tramo V, de margas grises. En este tramo se localiza el tránsito al Messiniense (Civis et al., 1990). Corresponden a la unidad estratigráfica Formación Écija, y solamente se observa fuera de la zona estudiada en este trabajo.

\section{FACTORES PALEOAMBIENTALES}

\subsection{Batimetría}

Durante la transgresión marina, mientras la masa de agua avanzaba hacia el interior del continente, la profundidad del mar fue aumentando. Como consecuencia de ello la batimetría del yacimiento fue variando en cada tramo estratigráfico. De acuerdo con la naturaleza de los sedimentos y la fauna que contienen se puede asignar cada tramo a diferentes pisos marinos (Fig. 9). Las arenas, gravillas y conglomerados (Tramos I y II) corresponden al piso infralitoral somero. En él habitaban grandes Crassostrea gryphoides, formando bancos, que pudieron 


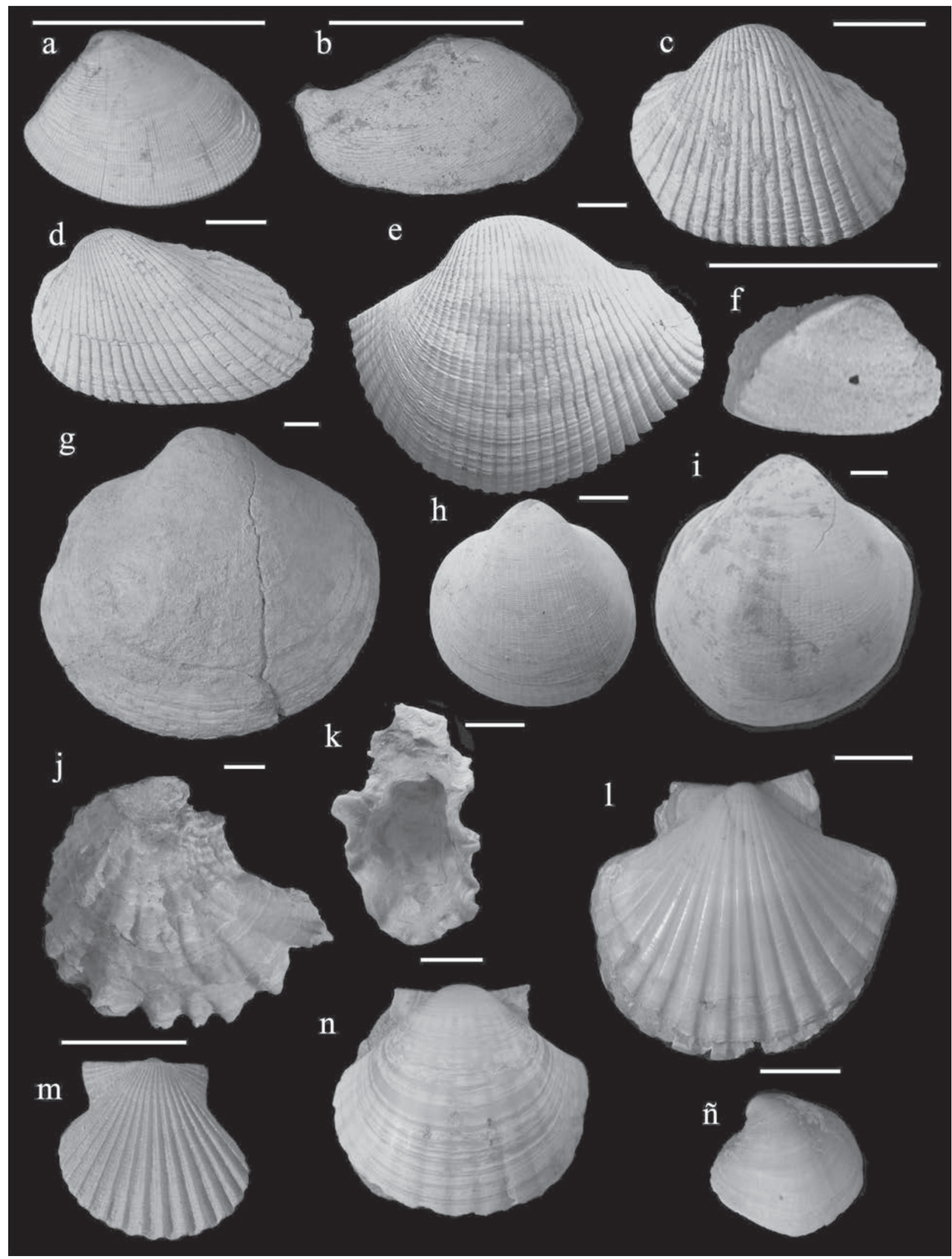

Figura 6. a) Nucula sulcata valva derecha. b) Nuculana pella valva derecha. c) Anadara diluvii valva Izquierda. d) Anadara pectinata valva izquierda. e) Anadara fichteli valva izquierda. f) Basterotia corbuloides valva derecha. g) Glycymeris bimaculata valva izquierda. h) Glycymeris insubrica valva izquierda. i) Glycymeris inflata valva derecha. j) Cubitostrea digitalina valva izquierda. k) Saccostrea virleti valva izquierda. l) Flabellipecten fraterculus valva derecha. m) Aequipecten radians valva izquierda. n) Oppenheimopectecten revolutus valva derecha. ñ) Megaxinus transversus valva izquierda. Escala de las barras $=10 \mathrm{~mm}$, excepto para (f) donde la barra $=5 \mathrm{~mm}$. 


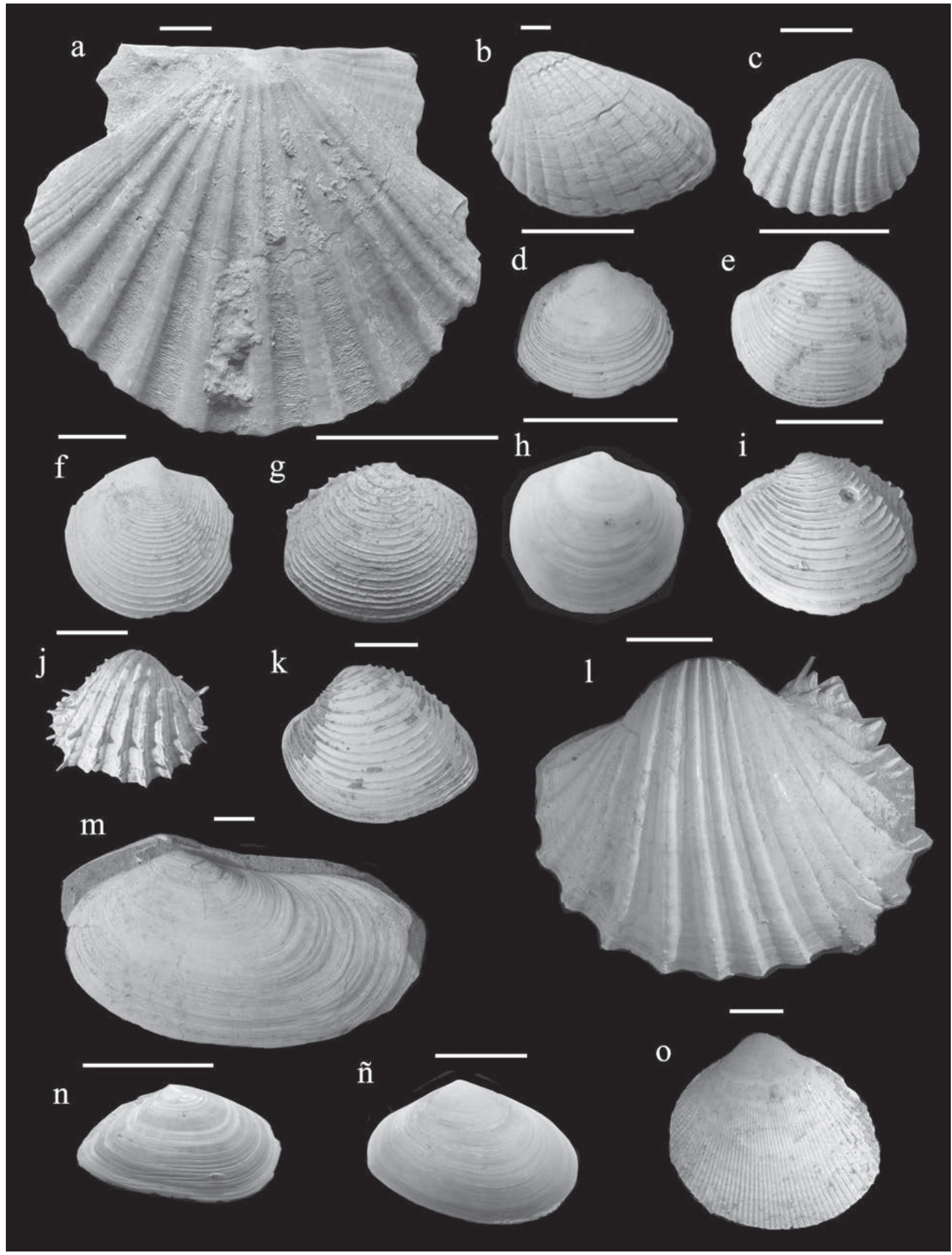

Figura 7. a) Gigantopecten albinus valva izquierda. b) Megacardita jouanneti valva izquierda. c) Centrocardita aculeata valva derecha. d) Gonimyrtea meneghinii valva derecha. e) Linga columbela valva izquierda. f) Lucinoma borealis valva derecha. g) Myrtea spinifera valva derecha. h) Diplodonta rotundata valva derecha. i) Lucina orbicularis valva izquierda. j) Acanthocardia paucicostata valva derecha. k) Venus nux valva izquierda. l) Cardium indicum valva izquierda. m) Lutraria oblonga valva izquierda. n) Tellina compressa valva derecha. ñ) Macoma elliptica valva derecha. o) Europicardium multicostatum valva izquierda. Escala de las barras $=10 \mathrm{~mm}$. 


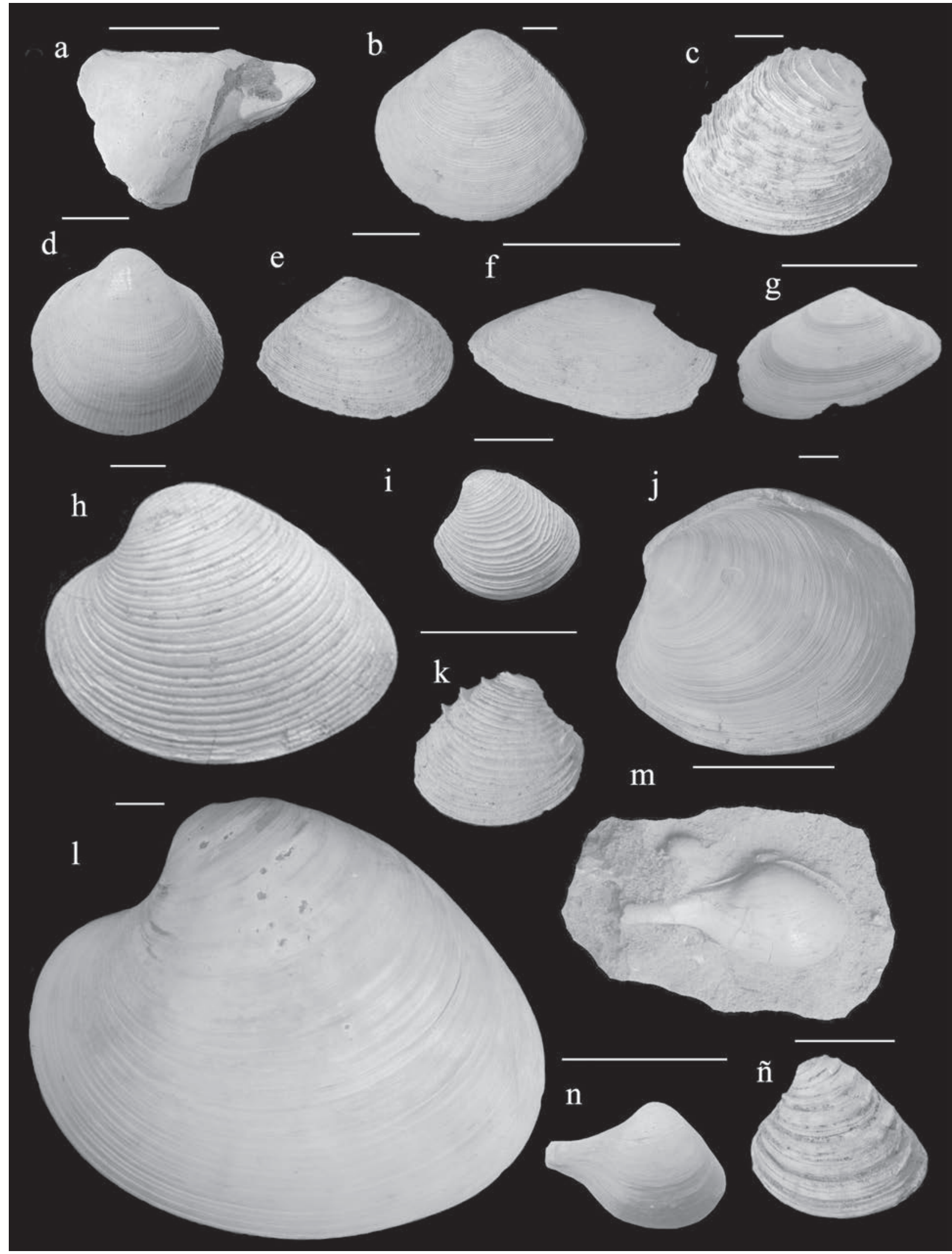

Figura 8. a) Pteria phalaenacea valva derecha. b) Arcopagia ventricosa valva izquierda. c) Circumphalus foliaceolamellosus valva izquierda. d) Laevicardium subturgidum valva izquierda. e) Tellina serrata valva derecha. f) Tellina distorta valva derecha. g) Tellina donacina valva izquierda. h) Callista erycinoides valva izquierda. i) Chamelea lamellosa rhysalea valva izquierda. j) Pelecyora brocchii valva izquierda. k) Clausinella dertoparva valva derecha. I) Callista italica valva izquierda. m) Cuspidaria rostrata valva derecha. n) Cuspidaria cuspidata valva derecha. ñ) Clausinella scalaris valva izquierda. Escala de las barras $=10 \mathrm{~mm}$. 
desarrollarse desde la "zona intertidal" (Harzhauser et al., 2016) hasta profundidades cercanas a $35 \mathrm{~m}$ (Kauffmann, 1969). Sobre ellas, y algo más profundas, se depositaron las arenas limosas (Tramo III) donde se acumularon gran cantidad de conchas pertenecientes a un elevado número de especies. De ellas, más del $75 \%$ habitaban en el piso infralitoral medio-profundo al que corresponde este tramo. Por encima de las anteriores, en un ambiente de fondo más tranquilo, se acumularon las margas areno-arcillosas (Tramo IV) con abundante bioturbación y especies del piso circalitoral interno, a una profundidad comprendida entre los 40 y $80 \mathrm{~m}$. Los materiales del Tramo V, no estudiados en este trabajo, podrían corresponder a los pisos circalitoral externo-batial interno (Fig. 9).

\subsection{Salinidad}

La elevada diversidad de especies de moluscos en la zona indica aguas con condiciones de salinidad normal. Esta idea queda reforzada por la presencia de especies de bivalvos estenohalinos como Saccella conmutata, Dosinia lupinus, Nuculana pella, Papilicardium papillosum y Acanthocardia paucicostata, todas ellas son frecuentes, e incluso muy abundantes, en el yacimiento. De entre estas, Acanthocardia paucicostata no tolera una salinidad fuera de los parámetros normales (Brambilla, 1976; Andrés, 1982) y Dosinia lupinus, en la actualidad, requiere una salinidad comprendida entre 28-30\% según algunos autores (Poppe \& Goto, 1993), o ligeramente superior como demuestra su presencia común en las costas andaluzas (Gofas et al., 2011), cuya salinidad supera el $30 \%$.

\subsection{Temperatura}

Son varios los factores que hacen pensar en una temperatura cálida para toda la zona durante el Tortoniense superior. La propia transgresión marina en la Cuenca del Guadalquivir fue causada, en parte o en su totalidad, por un ascenso eustático debido a una elevación de la temperatura global enmarcado dentro del ciclo 3.2 de Haq et al. (1987). Otro factor que refuerza la idea de temperatura cálida viene dado por la concentración del macroforaminífero Heterostegina gomez-angulensis, cuyo hábitat suele ser aguas cálidas (Tosquella et al., 2003), Además, la presencia de moluscos estenotérmicos de aguas cálidas, destacando entre los bivalvos especies tropicales o subtropicales como Circomphalus foliaceolamellosus, Cardium indicus, Callista erycinodes, C. italica, Linga columbela, Europicardium multicostatum, Gari uniradiata y otras especies tales como Tugonia anatina y Leporimetis lacunosa, que si bien no han sido localizadas en el yacimiento estudiado, si están presente en otros muy cercanos pertenecientes al CBT (Freneix et al., 1987b; Lauriat-Rage et al., 1999; Santos \& Mayoral, 2007). Entre los gasterópodos son muchas las especies de hábitos tropicales localizadas en el yacimiento como las correspondientes a las familias Ficidae, Olividae, Mitridae, Conidae y Terebridae entre otras (Landau et al., 2007).

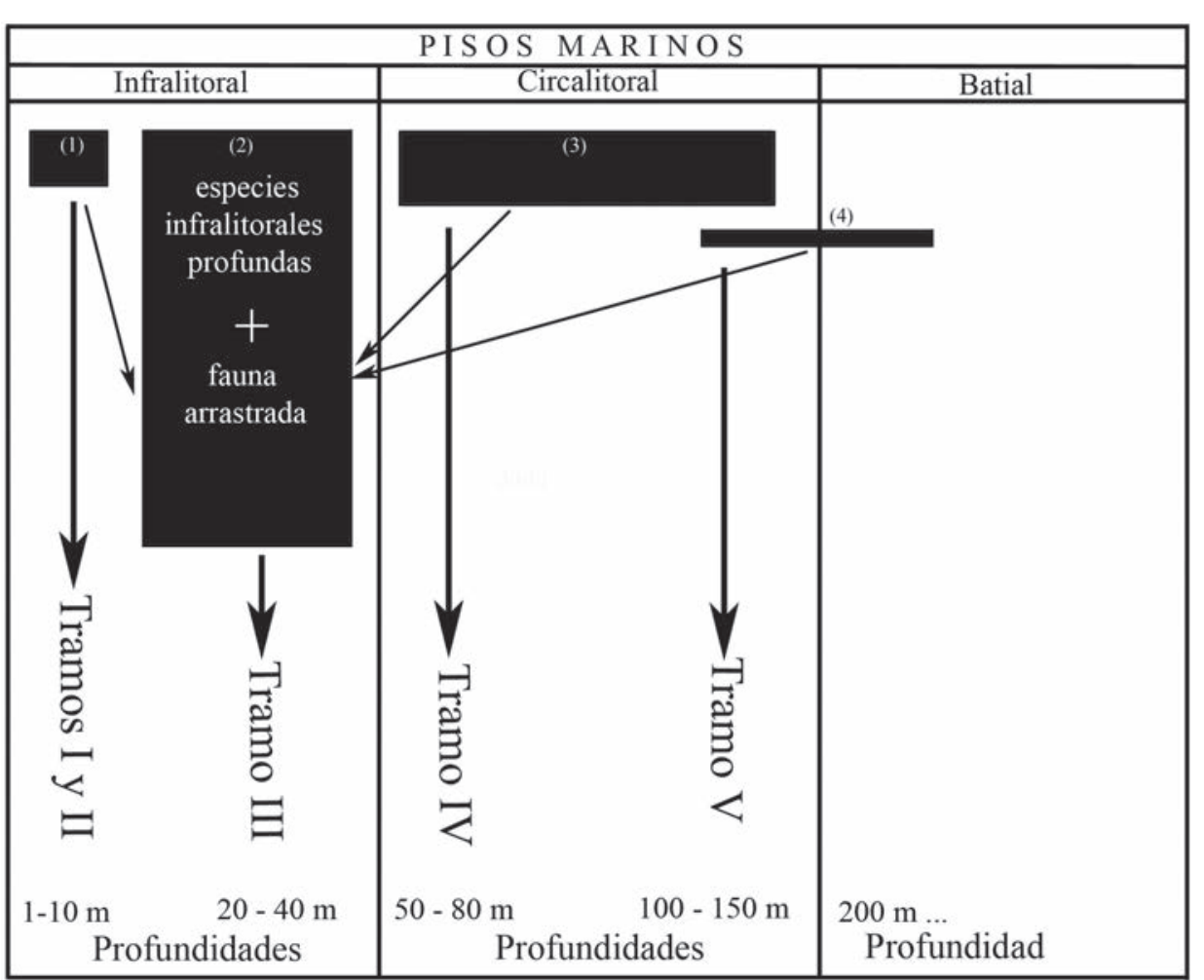

Figura 9. Los diferentes tramos estratigráficos del yacimiento, su correspondencia con los ambientes marinos y su batimetría. Los rectángulos negros representan las comunidades de bivalvos: (1): Infralitoral somero; (2): Infralitoral profundo; (3): Circalitoral; (4): Circalitoral profundo-Batial. Las flechas finas indican la procedencia de las especies arrastradas hacia el ambiente infralitoral profundo. 


\section{CONCLUSIONES}

Los movimientos de tierra realizados en la zona de estudio durante las últimas décadas han aportado nueva información y han permitido realizar una nueva columna estratigráfica a partir de dos secciones diferentes en afloramientos próximos. Se han establecido cuatro tramos en los materiales basales, los cuales corresponden al Complejo Basal Transgresivo (CBT). Dichos tramos registran un progresivo aumento batimétrico de muro a techo de la serie. No obstante, la presencia de paleocanales en el tramo III y la intercalación de un nivel de conglomerados y arenas, con restos de pectínidos y ostreidos, a techo del tramo IV sugieren una repentina y débil bajada del nivel del mar y posterior recuperación. El tramo III, formado por arenas limosas, corresponde al piso infralitoral externo con una profundidad de 20 a $40 \mathrm{~m}$. En él se localiza la mayor cantidad de especies fósiles, parte de las cuales han sido arrastradas desde los pisos limítrofes. La concentración de la fauna fósil en niveles, la ausencia de bioturbación en el sedimento y la falta de bioincrustaciones en las conchas son indicadores de fuerte agitación del medio y un rápido enterramiento de los restos existentes en dicho tramo. Dicho enterramiento se realizaba, posiblemente, de formas intermitentes, en épocas de tormentas.

Han sido identificados 132 taxones de bivalvos. De ellos, 125 se han clasificado a nivel de especie y 10 son citados por primera vez en la Península Ibérica. En un tercio de las muestras de sedimento estudiadas se han realizado conteos de los bioclastos comprobándose que el $58 \%$ pertenecen a bivalvos, el $32 \%$ a gasterópodos y el $10 \%$ restante a otros grupos. Los resultados del análisis taxonómico de las especies y familias confirman las similitudes entre las comunidades de bivalvos de Arroyo Trujillo y la del cercano yacimiento de Cacela (Portugal). En ambas coinciden las cinco familias más representadas en número de especies. Ambos yacimientos comparten, al menos, 76 especies (60,8\%), y el índice de Sorensen señala un $69 \%$ de similitud. La comunidad de bivalvos estudiada en el yacimiento indica un ambiente de salinidad normal y condiciones de temperatura tropical a subtropical.

\section{AGRADECIMIENTOS}

Los autores agradecen la ayuda inestimable prestada por Francisco González Portillo en la parte gráfica de este trabajo. También agradecemos a los revisores José Ángel González Delgado y otro anónimo sus comentarios y recomendaciones que sin duda han mejorado considerablemente el presente trabajo.

Este trabajo está dedicado a la memoria del Dr. Jorge Civis Llovera, recientemente fallecido. Formó parte del grupo de investigadores que nos precedió en el estudio del yacimiento Arroyo Trujillo.

\section{BIBLIOGRAFÍA}

Abad, M. 2002. Arquitectura Estratigráfica y Paleontológica del CBT. Universidad de Huelva, 249.

Abad, M. 2007. La Transgresión Tortoniense en el Margen Pasivo de la Cuenca del Guadalquivir: Respuesta Estratigráfica e Implicaciones Paleontológicas. Tesis Doctoral, Universidad de Huelva.

Acuña, J.D. 1978. Malacología del Mioceno marino de la provincia de Valencia. Estudios Geológicos, 34, 281-291.

Allen, J.A. \& Morgan, R. 1981. The functional morphology of the Atlantic deep water species of the families Cuspidariidae and Poromyidae (Bivalvia). An analysis of the evolution of the eptibranh condition. Philosophical transaction of the Royal Society of London. Serie B, 294, 413-546.

Andrés, I. 1982. Estudio Malacológico (Clase Bivalvia) del Plioceno Marino de Bonares (Huelva). Tesis Doctoral, Universidad de Salamanca. 1- 410.

Arias de Olivarrieta, J. 1918. Excursiones por el Plioceno de Cantillana (Sevilla) y cuenca del Biar. Boletín Real Sociedad Española de Historia Natural, 18, 523-529.

Baceta, J.I. \& Pendón, J.G. 1999. Estratigrafía y arquitectura de facies de la Formación Niebla, Neógeno superior, sector occidental de la Cuenca del Guadalquivir. Revista Sociedad Geológica España, 12, 419-438.

Bouchet, P. \& Rocroi, J. 2010. Nomenclator of Bivalve Familie; with a classification of bivalve families by $\mathrm{R}$. Bieler, J. G. Carter \& E. V. Coan. Malacologia, 52, 1-184.

Brambilla, G. 1976. I Molluschi Pliocenini di Villalvernia (Alessandria). I. Lamellibranchi. Memorie della Societa Italiana di Scienze Naturali e del Museo Civico di Storia Naturale Milano, 21, 81-128.

Brunetti, M.M. \& Vecchi, G. 2005. Rissoa quarantellii, una nuova specie del Pleistocene inferiores italiano. Bolletino Malacologico, 41, 60-65.

Cachao, M. 1995. Utilización de Nanofósseis Calcários em Biostratigrafia, Paleoceanografia e Paleoecología. Ampliaciones ao Noegénico do Algarve (Portugal) e do Mediterráneo Occidental (ODP 653) e á Problemática do Coccolithus pelagicus. Ph.D. Thesis. Facultade de Ciencias da Universidade de Lisboa, 1-356 (inédito).

Cerulli-Irelli, S. 1908. Fauna Malacologica Mariana 2a parte. Paleontographia Italica, vol. XIV, 8-64.

Civis, J., Sierro, F.J., González Delgado, J.A., Flores, J.A., Andrés, I., Porta, J. \& Valle, M.F. 1987. El Neógeno marino de la provincia de Huelva: antecedentes y definición de las unidades litográficas. In: Paleontología del Neógeno de Huelva (ed. Civis, J.). Universidad de Salamanca. 9-21.

Civis, J., González Delgado, J.A., Andrés, I., Valle, M.F., Sierro, F.J., Flores, J.A. \& Dabrio, C.I. 1990. El contenido paleontológico de la sección Tortoniense-Messiniense de Arroyo Trujillo (Sevilla, borde NW de la Cuenca del Guadalquivir). Actas de Paleontología, 68, 85-101.

Cossmann, M. \& Peyrot, A. 1911. Conchologie néogénique de 1'Aquitaine. II. Pélécypodes. Actes de la Société Linnéenne de Bordeaux, 65, 51-333. 
Freneix, S., Saint Martin, J.P. \& Moissette, P. 1987. Bivalves Hetérodontes du Messinian d'Oraine (Algérie occidentale). Bulletin du Muséum National d'Historie Naturalle, 4e ser., section $C, 9,414-453$.

Gavala, J. 1927. Mapa Geológico de España. Memoria de la Hoja de Cantillana. Boletín del I.G.M.E. Serie 3, XLIXX (IX), 5-79.

Gofas, S., Moreno, D. \& Salas, C. (Coordinadores). 2011. Moluscos marinos de Andalucía, II. Servicio de Publicaciones e Intercambio Cientifico. Universidad de Málaga, 343-798.

González Delgado, J.A. \& Civis, J. 2000. Ciclicidad estacional en perfiles de isótopos de $\mathrm{C}$ y $\mathrm{O}$ de Megacardita jouannti del Tortoniense superior de Cacela (Algarve, Portugal y Arroyo Trujillo (Sevilla España). Ciencias da Terra, 14, 297-302.

González Delgado, J.A., Andrés, I. \& Sierro. F.J. 1995. Late Neogene molluscan faunas from the Northeast Atlantic (Portugal, Spain, Marocco). Geobios, 28, 4, 459-471; doi: 10.1016/S0016-6995(95)80021-2.

Haq, B.V., Hardenbol, J. \& Vail, P.R. 1987. Chronology of fluctuating sea levels since the Triassic. Science, 235, 1156-1167; doi: 10.1126/science.235.4793.1156.

Harzhauser, M., Djuricic, A., Mandic, O., Neubauer, T.A., Zuschin, M. \& Pfeifer, N. 2016. Age structure, carbonate production and shell loss rate in an Early Miocene reef of the giant oyster Crassostrea gryphoides. Biogeosciences, 13, 1223-1235; doi: 10.5194/bg-13-1223-2016.

Janssen, A.W. \& Van Der Slik, L. 1971. De fossiele schelpen van de Nederlandse stranden en zeegaten, tweede serie. $4^{1}$ ). Basteria, 35, 1-4.

Janssen, R. \& Kryloga, E.M. 2014. Deep-sea fauna of European seas: An annotated species check-list of benthic invertebrates living deeper than $2000 \mathrm{~m}$ in the seas bordering Europe. Bivalve. Invertebrate Zoology, $11,43-82$.

Kauffmann, E.G. 1969. Form, function and evolution. In: Treatise on Invertebrate Paleontology N. Vol. 1 Mollusca 6 Bivalvia (ed. Moore R.C.). Geological Society of America and University of Kansas Press, 129-205.

Kautsky, F. 1939. Die Erycinen des niederösterreichisschen Miocaen. Annalen des Naturhistorischen museo Wien, 51, 584-671.

Landau, B., Capelo J.C. \& Marques da Silva, C. 2007. Patterns of extinction and local disappearance of tropical marine gastropods; contrasting examples from across the north Atlantic. Açoreana, Sup 5, 50-58.

Lauriat-Rage, A., Ben Moussa, A., Piquet, J.P. \& Saint Martin, J.P. 1997. The bivalvia (Mollusca) from the upper Miocene of the Sais Basin (Southern Rifian corridor. Marocco). Palaeobiogeography and Palaeoecology. Revista Sociedad Geológica de España, 12, 77-84.

Lozano Francisco, M.C. 1997. Los Bivalvos del Plioceno de la Provincia de Málaga. Tesis Doctoral Universidad de Málaga., 1-734.

Malatesta, A. 1974 Malacofauna pliocenica Umbra. Memorie per serviré descrizione della Carta Geológica d'Italia, $13,1-498$.
Marasti, R. 1973. La fauna tortoniana de T. Stirone (limite Parmense-Piacentino). Bolletino della Societá Paleontologica Italiana, 12, 76-12

Martín, J.M., Braga, J.C. \& Betzler, C. 2001. The Messinian Guadalhorce Corridor: The last northern, AtlanticMediterranean Gateway. Terra Nova, 13, 418-424; doi: 10.1046/j1365-3121.2001.00376.x.

Martínez Graña, A.M., Bajo, I., González Delgado, J.A., Cárdenas Carretero, J., Abad, M. \& Legoinha, P. 2016. Entornos virtuales 3D aplicados al Patrimonio Paleontológico: Neógeno de Sevilla (Cuenca del Guadalquivir, España). Actas de la XXXII Jornadas de la Sociedad Española de Paleontología. Molina de Aragón, 201-206.

Martínez Lázaro, N. 2004. Lucinidae (Mullusca, Bivalvia) del Mioceno de la Cuenca del Vallés-Penedés (Cataluña, España). Pliocenica, 4, 16-35.

Millard, V. 1997. Classification of Mollusca: A Classification of Worldwide Mollusca. University of California, p. 544.

Mocho, P., Pereira, S. \& Lourenço, J. 2010. Bivalves marinhos do Miocénico superior, (Tortoniano inferior) de Foz de Rego (Costa de Caparica, Portugal). Revista Electrónica de Ciências da Terra. Geosciences On-Line Journal, 17, 1-4.

Nyst, P.H. 1878-1881. Conchyliologic des terrains tertiaries de la belgique. Annales du Museé Royal d'Histoire Naturalle de Belgique 3, 1-263.

Nyst, P.H. \& Wertendorp, G.D. 1839. Nouvelles recherches sur les coquilles fossils de la provincial d'Anvers. Bolletines de l'Académie Royal des Science y Bellasletras de Bruxelles, 6, 393-414.

Pendón, J.G., González Regalado, M.L., Ruiz, F., Abad, M. \& Tosquella, J. 2001. Pulsos transgresivos neógenos en el sector central de la Cuenca del Guadalquivir. Geotemas, 3, 13-16.

Pereira da Costa, F. A. 1903-1904. Mollusques Tertiaires du Portugal. Planches de Céphalopodes, Gastéropodes et Pélécypodes. Commission du Service Géologique du Portugal, 1-45.

Poppe, G.T. \& Goto, Y. 1993 European Seashells (Scaphopoda, Bivalvia, Cephalopoda). Volume 2. Verlang Christa Hemmer, 1-221.

Robba, E. 1968. Molluschi del Tortoniano-Tipo (Piamonte). Rivista Italiana di Paleontología, 74, 457-646.

Sabelli, B., Giannuzzi-Zavelli, R. \& Bedulli, D. 19901992. Catalogo Annotato dei Molluschi Marini del Mediterraneo. Libreria Piani. Bologna.

Sacco, F. 1897-1898. I Molluschi dei terreni terziarii del Piamonte e della Liguria. XXIII, XXIV, XXV, XXVI, XXVII. Ed. Carlos Clausen. Libraio della Academia delle Scienze. Torino, 68, 76, 102.

Santos, A. \& Mayoral, E. 2007. Paleoecología de la malacofauna de bivalvos del Mioceno superior de Cacela (SE Portugal). Treballs del Museu de Geología de Barcelona, 15, 25-49.

Sanz de Galdeano, C. \& Vera, J.A. 1991. Una propuesta de clasificación de las cuencas neógenas béticas. Acta Geológica Hispánica, 26, 205-227. 
Sierro, F.J. 1985. The replacement of the "Globorotalia menardi" group by the "Globorotalia miotumida" group: an aid to recognizing the Tortonian-Messinian boundary in the Mediterranean and adjacent Atlantic. Marine Micropaleontology, 9, 525-535.

Studencka, B. 1986. Bivalves from the Badenian (middle Miocene) marine sandy facies of Southern Poland. Acta Paleontologia Polonica, 47, 3-128.

Tosquella, J., Abad, M., González Regalado, M.L., Ruiz, F. \& Pendón, J.G. 2003. Paleoecología de los niveles lumaquélicos con Heterosteginas en el Tortoniense del sector central de la Cuenca del Guadalquivir (S. O. España). Geogaceta, 33, 23-36.

Venzo, S. \& Pelosio, G. 1963. La malacofauna tortoniana del Colle di Vigoleno. (Preappenino Piacentino). Paleontographia Italica, 58, 43-213.
Verdenius, J.G. 1970. Neogene Stratigraphy of the Western Guadalquivir Basin (Southern Spain). Utrecht Micropaleontological Bulletins, 3, 1-107.

Wagner, R.H. \& Álvarez-Vázquez, C. 2010. The Carboniferous floras of the Iberian Peninsula: A synthesis with geological connotations. Review of Palaeobotany and Palynology, 162, 238-324; doi: 10.1016/j.revpalbo.2010.06.005.

Wenz, W. 1942. Eine Vindobon-fauna aus der Umgebung von Cantillana. Prov. Sevilla. Spanien. Natur Museum Senckenberg. 25, 207-221.

Wesselingh, F. \& Moerijk, P. 2007. Fossielenatlas Nieuwsbrief, Netherlands Malacogische Vereniging, 19, 1-8. 